\title{
PPRA / PCMSO: Auditoria, Inspeção do Trabalho e Controle Social
}

Carlos Roberto Miranda'

Carlos Roberto Dias ${ }^{2}$

\author{
Environmental Risk Prevention \\ Programs / Occupational Health \\ Medical Control Programs: Audits, \\ Employment Inspection and \\ Social Control
}

'Médico do Trabalho, Mestre em Saúde Comunitária, Auditor Fiscal do Trabalho. ${ }^{2}$ Médico do Trabalho, Pós-graduado em Higiene Ocupacional, Auditor Fiscal do Trabalho.
Os autores analisaram Programas de Prevenção de Riscos Ambientais (PPRA) e Programas de Controle Médico de Saúde Ocupacional (PCMSO) implementados por 30 empresas, de diferentes ramos econômicos, com mais de 100 (cem) empregados, em atividade em Salvador, Bahia. As inconsistências verificadas foram estudadas segundo os riscos ocupacionais, levando-se em conta as diversas etapas de desenvolvimento dos programas. Os autores constataram a baixa qualidade técnica desses programas e apontam a evidente necessidade de ampliar a cobertura da fiscalização estatal, assim como de estimular a participação dos trabalhadores e dos seus representantes no desenvolvimento dos programas PPRA e PCMSO. Consideram, ainda, de fundamental importância o desenvolvimento e o aprimoramento de condutas, procedimentos e instrumentos de inspeção na área de segurança e saúde no trabalho.

Palavras-chaves Riscos Ambientais, Programas de Saúde Ocupacional, Auditoria, Inspeção do Trabalho.

The authors evaluated Environmental Risk Prevention / Occupational Health Medical Control Programs in use by 30 companies operating in various fields, each with over 100 employees, in the city of Salvador, State of Bahia, Brazil. The inconsistencies encountered were examined in light of the occupational risks involved, considering the various stages inherent to program development. The authors perceived the low degree of technical quality of the Programs in question and indicated the need for more widespread involvement of State Inspectors, plus the encouragement of workers and their representatives with regards the development of Environmental Risk Prevention / Occupational Health Medical Control Programs. Furthermore, they also considered the development and enhancement of codes of conduct, procedures and inspection instruments to be of prime importance for work safety and health.

Keywords Environmental Risks, Occupational Health Programs, Auditing, Employment Inspection. 


\section{Introdução}

A legislação brasileira que trata da segurança e da saúde no trabalho passou a adotar um novo enfoque, a partir do final de 1994, ao estabelecer a obrigatoriedade das empresas elaborarem e implementarem dois programas: um ambiental, o PPRA Programa de Prevenção de Riscos Ambientais, e outro médico, o PCMSO Programa de Controle Médico de Saúde Ocupacional. Adotando como paradigma a Convenção 161/85 da Organização Internacional do Trabalho (OIT), a legislação brasileira específica passou a considerar as questões incidentes não somente sobre o indivíduo mas, também sobre a coletividade de trabalhadores, promovendo, assim, uma ampliação do conceito restrito de "medicina do trabalho" $(1,2)$

Em verdade, apesar do Brasil ter ratificado em 1991 a Convenção 161 da OIT, até 1994 as Normas Regulamentadoras (NRs) caracterizavam-se ainda por um enfoque essencialmente "individualista". As NR-7 e 9 intitulavam-se, respectivamente, Exames Médicos e Riscos Ambientais, ou seja, a ênfase era, isoladamente, ora para o corpo do trabalhador, ora para a avaliação quantitativa de um certo risco ambiental. As novas normas, preocupadas agora com a saúde do conjunto dos trabalhadores, privilegiaram o instrumental clínicoepidemiológico na abordagem da relação saúde/trabalho e introduziram a questão da valorização da participação dos trabalhadores e do controle social. Neste sentido, a exigência legal dos novos programas PCMSO e PPRA representou, na prática, a superação de um "viés biologista/ ambiental" e a introdução de um "olhar coletivo" nas questões relacionadas com a segurança e a saúde dos trabalhadores brasileiros. $(3,4)$

\section{PPRA Programa de Prevenção de Riscos Ambientais}

O PPRA, cuja obrigatoriedade foi estabelecida pela NR-9 da Portaria 3214/ 78 , apesar de seu caráter multidisciplinar, é considerado essencialmente um programa de higiene ocupacional que deve ser implementado nas empresas de forma articulada com um programa médico - o PCMSO. $(5,6)$
Todas as empresas, independente do número de empregados ou do grau de risco de suas atividades, estão obrigadas a elaborar e implementar o PPRA, que tem como objetivo a prevenção e o controle da exposição ocupacional aos riscos ambientais, isto é, a prevenção e o controle dos riscos químicos, físicos e biológicos presentes nos locais de trabalho.

A NR-9 detalha as etapas a serem cumpridas no desenvolvimento do programa, os itens que compõem a etapa do reconhecimento dos riscos, os limites de tolerância adotados na etapa de avaliação e os conceitos que envolvem as medidas de controle. A norma estabelece, ainda, a obrigatoriedade da existência de um cronograma que indique claramente os prazos para o desenvolvimento das diversas etapas e para o cumprimento das metas estabelecidas.

Um aspecto importante deste programa é que ele pode ser elaborado dentro dos conceitos mais modernos de gerenciamento e gestão, onde o empregador tem autonomia suficiente para, com responsabilidade, adotar um conjunto de medidas e ações que considere necessárias para garantir a saúde e a integridade física dos seus trabalhadores.

A elaboração, implementação e avaliação do PPRA podem ser feitas por qualquer pessoa, ou equipe de pessoas que, a critério do empregador, sejam capazes de desenvolver o disposto na norma. Além disso, cabe à própria empresa estabelecer as estratégias e as metodologias que serão utilizadas para o desenvolvimento das ações, bem como a forma de registro, manutenção e divulgação dos dados gerados no desenvolvimento do programa.

As ações do PPRA devem ser desenvolvidas no âmbito de cada estabelecimento da empresa, sendo que sua abrangência e profundidade dependem das características dos riscos existentes no local de trabatho e das respectivas necessidades de controle.

A NR-9 estabelece as diretrizes gerais e os parâmetros mínimos a serem observados na execução do programa mas os mesmos podem ser ampliados mediante negociação coletiva de trabalho. Procurando garantir a efetiva implementação do PPRA, a norma estabelece que a empresa deve 
adotar mecanismos de avaliação que permitam verificar o cumprimento das etapas, das ações e das metas previstas. Além disso, a NR-9 prevê algum tipo de controle social, garantindo aos trabalhadores o direito à informação e à participação no planejamento e no acompanhamento da execução do programa.

\section{PCMSO Programa de Controle Mé- dico de Saúde Ocupacional}

O PCMSO, cuja obrigatoriedade foi estabelecida pela NR-7 da Portaria 3214/ 78, é um programa médico que deve ter caráter de prevenção, rastreamento e diagnóstico precoce dos agravos à saúde relacionados ao trabalho. Entende-se aqui por "diagnóstico precoce", segundo o conceito adotado pela Organização Mundial da Saúde (OMS), a detecção de distúrbios dos mecanismos compensatórios e homeostáticos, enquanto ainda permanecem reversíveis alterações bioquímicas, morfológicas e funcionais.

Todas as empresas, independente do número de empregados ou do grau de risco de sua atividade, estão obrigadas a elaborar e implementar o PCMSO, que deve ser planejado e implantado com base nos riscos à saúde dos trabalhadores, especialmente os riscos identificados nas avaliações previstas no Programa de Prevenção de Riscos Ambientais (PPRA). Entre suas diretrizes, uma das mais importantes é aquela que estabelece que o PCMSO deve considerar as questões incidentes tanto sobre 0 indivíduo como sobre a coletividade de trabalhadores, privilegiando o instrumental clínico-epidemiológico.

A norma estabelece, ainda, o prazo e a periodicidade para a realização das avaliações clínicas, assim como define os critérios para a execução e interpretação dos exames médicos complementares los indicadores biológicos).

Em síntese, na elaboração do PCMSO, o mínimo requerido é um estudo prévio para reconhecimento dos riscos ocupacionais existentes na empresa, através de visitas aos locais de trabalho, baseando-se nas informações contidas no PPRA. A partir deste reconhecimento de riscos, deve ser estabelecido um conjunto de exames clínicos e complementares específicos para cada grupo de trabalhadores da empresa, utilizan- do-se de conhecimentos científicos atualizados e em conformidade com a boa prática médica. Assim, o nível de complexidade do PCMSO depende basicamente dos riscos existentes em cada empresa, das exigências físicas e psíquicas das atividades desenvolvidas e das características biopsicofisiológicas de cada população trabalhadora. (7) A norma estabelece as diretrizes gerais e os parâmetros mínimos a serem observados na execução do programa, podendo os mesmos, entretanto, ser ampliados mediante negociação coletiva de trabalho.

O PCMSO deve ser coordenado por um médico, com especialização em medicina do trabalho, que será o responsável pela execução do programa. Ao empregador, por sua vez, compete garantir a elaboração e efetiva implementação do PCMSO, bem como zelar pela sua eficácia. Procurando garantir a efetiva implementação do PCMSO, a NR-7 estabelece que o programa deverá obedecer a um planejamento em que estejam previstas as ações de saúde a serem executadas durante $o$ ano, devendo estas ser objeto de relatório anual. O relatório anual deverá discriminar, por setores da empresa, o número e a natureza dos exames médicos, incluindo avaliações clínicas e exames complementares, estatísticas de resultados considerados anormais, assim como o planejamento para o ano seguinte.

\section{A inspeção do trabalho e os pro- gramas PPRA/PCMSO}

A inseparabilidade entre o trabalho e o indivíduo que o realiza, a implicação da pessoa do trabalhador na atividade laboral, determinam uma exigência de tutela de sua liberdade e integridade física, ou seja, em última instância determinam a intervenção do Estado na regulamentação das relações de trabalho. Em conseqüência, na medida em que o trabalho é de alguma forma normatizado, a inspeção encontra sentido e lugar de ser na história do trabalho. Em síntese, o serviço de inspeção deveria ser a forma de tornar efetivas as regulamentações do processo de trabaIho. (8)

Entretanto, não são poucas as dificuldades relacionadas à inspeção e ao controle dos ambientes de trabalho. A complexida- 
de cada vez maior das relações trabalhistas exige que o Auditor do Trabalho tenha uma boa formação jurídica e técnica. O caráter multidisciplinar da inspeção do trabalho justifica a incorporação de carreiras técnicas que aportem ao sistema de inspeção e proteção do trabalho os conhecimentos teóricos e práticos que são necessários para atender adequadamente as questões que se relacionam com a segurança e saúde dos trabalhadores. (4)

Neste sentido, a Convenção n 81 da OIT adotada em 1947 e ratificada pelo Brasil em 1957, estabelece em seu artigo 10 que o número de inspetores de trabalho deve ser o suficiente para permitir o exercício eficaz das funções de serviço de inspeção e será fixado tendo-se em conta o número, a natureza, a importância e a situação dos estabelecimentos sujeitos ao controle da inspeção, assim como o número e a diversidade das categorias de trabalhadores ocupados nesses estabelecimentos. (9)

Em nosso país, o Ministério do Trabalho e Emprego - MTE é o órgão de âmbito nacional competente para coordenar, orientar, controlar e supervisionar as atividades relacionadas com a segurança e saúde no trabalho, inclusive a fiscalização do cumprimento dos preceitos legais e regulamentares, em todo o Território Nacional. A nível estadual, essa fiscalização é executada pelas Delegacias Regionais do Trabalho. Para desenvolver a fiscalização na área de segurança e saúde no trabalho em todo - país, o MTE dispõe atualmente de 690 Auditores fiscais. Vale observar que esse contingente é claramente insuficiente para inspecionar um total de mais de 4 milhões de estabelecimentos em atividade e dar cobertura para uma população economicamente ativa hoje em torno de 28 milhões de trabalhadores. Esses dados revelam a existência de um auditor fiscal para cerca de 6.000 estabelecimentos, ou seja, um auditor fiscal para cada 40.000 trabalhadores em atividade no país.

No caso do Estado da Bahia o quadro é ainda mais grave pois a Delegacia Regional do Trabalho dispõe de apenas 20 auditores na área de segurança e saúde no trabalho $(11$ médicos do trabalho e 9 engenheiros de segurança) para inspecionar cerca de 200.000 estabelecimentos em atividade no Estado e dar cobertura para uma população economicamente ativa hoje em torno de 1.300.000 trabalhadores. Ou seja, um auditor fiscal para cerca de 10.000 estabelecimentos, ou, um auditor fiscal para cada 65.000 trabalhadores em atividade. $(10,11)$

Em relação ao PPRA, do ponto de vista da inspeção do trabalho, certos procedimentos obrigatórios previstos na NR-9 podem permitir um melhor acompanhamento do programa. Um aspecto fundamental é a obrigatoriedade do empregador reconhecer os riscos ambientais presentes nos diversos locais de trabalho da empresa e assumir prazos para solucionar as questões relativas a esses riscos. Como o programa é permanente, cabe ao empregador formalizar um cronograma anual, com estabelecimento das ações a serem executadas e as metas a serem alcançadas neste período. Por sua vez, a exigência da manutenção de um histórico com o registro dos dados mantido por um período mínimo de vinte anos permite aos Auditores Fiscais do Trabalho verificar e comprovar tecnicamente os resultados alcançados no desenvolvimento do programa.

Quanto ao PCMSO, ao estabelecer a obrigatoriedade de um planejamento em que estejam previstas as ações de saúde a serem executadas durante $o$ ano, as quais devem ser objeto de um relatório anual, a NR-7 permitiu também um melhor acompanhamento do programa médico da empresa.

\section{Metodologia}

O presente trabalho teve como objetivo principal auditar, do ponto de vista da inspeção do trabalho, Programas de Prevenção de Riscos Ambientais (PPRA) e Programas de Controle Médico de Saúde Ocupacional (PCMSO) elaborados e implementados por empresas, de diferentes ramos econômicos, com mais de cem empregados, em atividade em Salvador, Bahia. Além disso, buscou-se avaliar a participação dos trabalhadores e dos seus representantes no desenvolvimento desses programas e, ao mesmo tempo, definir e aprimorar condutas, procedimentos e instrumentos de inspeção na área de segurança e saúde no trabalho.

Foram utilizados dados primários e secun- 
dários. Os dados primários foram coletados pelos próprios autores, dois Auditores Fiscais (Médicos do Trabalho) lotados na Delegacia Regional do Trabalho na Bahia, no período entre abril e dezembro de 2002, utilizando um questionário ("checklist") especificamente elaborado para levantamento dos riscos ambientais presentes nos locais de trabalho. Para efeito deste trabalho, foram considerados "riscos ambientais", os agentes químicos, físicos e biológicos existentes nos ambientes de trabalho que, em função de sua natureza, concentração ou intensidade e tempo de exposição, são capazes de causar danos à saúde do trabalhador, conforme conceituação adotada pela NR-9, item 9.1.5, da Portaria 3214/78. Os dados secundários, por sua vez, foram obtidos a partir dos documentos-base, dos históricos e dos relatórios anuais dos Programas de Prevenção de Riscos Ambientais (PPRA) e dos Programas de Controle Médico de Saúde Ocupacional (PCMSO) elaborados e implementados por empresas privadas de diferentes ramos econômicos, com mais de 100 (cem) empregados, em atividade em Salvador, Bahia. Os programas foram analisados e auditados, tomando como base a identificação e o reconhecimento dos riscos ambientais, assim como a avaliação do cumprimento das etapas, das ações e das metas previstas em seus documentos-base e respectivos relatórios anuais. As análises incluíram, ainda, as estratégias e as metodologias utilizadas pelas empresas para o desenvolvimento de seus respectivos programas.

\section{A amostra}

Foram inspecionadas 30 empresas com mais de cem empregados e em atividade em Salvador, Bahia. Cumpre observar que essa amostra representa 6,5\% das empresas com mais de cem empregados, em atividade em Salvador, nos setores da indústria, comércio e serviços. De acordo com consulta parametrizada no SFIT - Sistema Federal de Inspeção do Trabalho, durante o ano de 2002, na cidade de Salvador, nos referidos setores, estavam em atividade 465 empresas com mais de cem empregados (12).

\section{Empresas inspecionadas}

Das 30 empresas inspecionadas, 10 $(33,3 \%)$ delas pertencem ao setor Industrial, $10(33,3 \%)$ ao setor de Serviços e 10 $(33,3 \%)$ ao setor de Comércio.

Em relação ao grau de risco da atividade econômica desenvolvida, 11 (36,7\%) das empresas foram classificadas como grau de risco dois, $17(56,7 \%)$ como grau de risco três e $2(6,7 \%)$ como grau de risco quatro, de acordo com o Quadro I da NR4 da Portaria 3214/78.

Quanto ao número de empregados, todas as empresas selecionadas mantém mais de 100 trabalhadores em atividade, sendo que $15(50,0 \%)$ delas possuem entre 100 e 200 empregados, $4(13,3 \%)$ entre 201 e 300 empregados, $4(13,3 \%)$ entre 301 e 400 empregados, $2(6,7 \%)$ entre 401 e 500 empregados e $5(16,7 \%)$ possuem mais de 500 empregados.

\section{Resultados}

\section{PPRA: resultado das auditorias}

Os Programas de Prevenção de Riscos Ambientais (PPRA) foram analisados, tomando-se como base a identificação e o reconhecimento dos riscos ambientais existentes nos locais de trabalho, assim como a avaliação do cumprimento das etapas, das ações e das metas previstas em seus documentos-base e respectivos relatórios anuais. Os resultados são apresentados na Tabela 01 , tendo sido analisados os seguintes tópicos:

\section{a. Elaboração do programa}

Todas as empresas, independente do número de empregados ou do grau de risco de suas atividades, estão obrigadas a elaborar e implementar o PPRA, de acordo com a NR-9, item 9.1.1, da Portaria 3214/ 78. Das 30 empresas inspecionadas, 2 $(6,7 \%)$ não tinham elaborado e implementado o referido programa.

\section{b. Responsabilidade técnica}

De acordo com a NR-9, item 9.3.1.1, a elaboração e implementação do PPRA poderão ser feitas por qualquer pessoa, ou equipe de pessoas que, a critério do em- 
Tabela 01 PPRA: Resultados das Auditorias.

\begin{tabular}{|l|c|c|}
\hline Procedimentos & $\begin{array}{c}\text { Sim } \\
\%\end{array}$ & $\begin{array}{c}\text { Não } \\
\%\end{array}$ \\
\hline Elaboração do PPRA & 93,3 & 6,7 \\
\hline Avcliação anual do PPRA & 14,3 & 85,7 \\
\hline Inconsistências no PPRA & 92,9 & 7,1 \\
\hline Inconsistências relacionados com riscos físicos & 82,1 & 17,9 \\
\hline Inconsistências relacionados com riscos químicos & 28,6 & 71,4 \\
\hline Inconsistências relacionados com riscos biclógicos & 0,0 & 100,0 \\
\hline Inconsistências no planejamento das ações & 10,7 & 89,3 \\
\hline Inconsistências no reconhecimentos dos riscos ambientais & 42,9 & 57,1 \\
\hline Inconsistências na avaliação quantitativa dos riscos & 39,3 & 60,7 \\
\hline Inconsistências na implementação de medicas coletivas & 71,4 & 28,6 \\
\hline Inconsistências no cronoçrama de execuçc̃o das ações & 71,4 & 28,6 \\
\hline
\end{tabular}

pregador, sejam capazes de desenvolver o disposto na norma. Entre as 28 empresas inspecionadas que elaboraram o PPRA, em $14(50,0 \%)$ delas o programa tinha sido elaborado por um engenheiro de segurança do trabalho, em $13(46,4 \%)$ por um técnico de segurança do trabalho e em 1 $(3,6 \%)$ por um médico do trabalho. Apesar de ser considerado essencialmente um programa de higiene ocupacional, em nenhuma das empresas o programa tinha sido elaborado por um profissional higienista ocupacional.

\section{c. Avaliação anual}

A norma estabelece que a empresa deve adotar mecanismos de avaliação que permitam verificar o cumprimento das etapas, das ações e das metas previstas. De acordo com a NR-9, item 9.2.1.1, uma avaliação global do PPRA deve ser efetuada, sempre que necessário e pelo menos uma vez ao ano, para a avaliação do seu desenvolvimento e realização dos ajustes necessários e estabelecimento de novas metas e prioridades. Entre as 28 empresas inspecionadas que elaboraram o PPRA, 24 $(85,7 \%)$ não tinham efetuado pelo menos uma avaliação anual do seu programa.

\section{d. Inconsistências identificadas}

Entende-se aqui por "inconsistência", a qualidade ou estado de falta de consistência, de fundamento ou de coerência entre os dados e informações contidas nos documentos fornecidos pela empresa e aqueles verificados fisicamente no local de trabalho pelos Auditores Fiscais do Trabalho. Neste sentido, foram avaliadas as seguintes etapas do PPRA: reconhecimento dos riscos ambientais, estabelecimento de prioridades $\mathrm{e}$ metas de avaliação e controle, avaliação quantitativa dos riscos e da exposição dos trabalhadores e, implantação de medidas de controle e avaliação de sua eficácia.

Entre as 28 empresas que elaboraram o PPRA, $26(92,9 \%)$ delas apresentaram algum tipo de inconsistência em seu programa. Analisando-se as empresas que apresentaram inconsistências segundo as etapas do programa, em 12 (42,9\%) delas a inconsistência referia-se ao reconhecimento dos riscos, em $11(39,3 \%)$ à avaliação quantitativa, em $20(71,4 \%)$ à implantação de medidas coletivas, em 3 (10,7\%) ao planejamento do programa e, em 20 $(71,4 \%)$ a inconsistência estava relacionada ao cronograma de execução das ações.

Estudando-se as inconsistências segundo os riscos ocupacionais, verificou-se que em $82,1 \%$ dos casos as inconsistências relacionavam-se com os riscos físicos, em $28,6 \%$ essa relação era com os riscos químicos e, em nenhum caso as inconsistências estavam relacionadas com os riscos biológicos presentes nos locais de trabalho.

No caso das empresas que apresentaram inconsistências relacionadas com os riscos químicos, em 7,1\% delas a inconsistência referia-se ao reconhecimento dos riscos, em $35,7 \%$ à avaliação quantitativa, em $25,0 \%$ à implantação de medidas coletivas e, em $7,1 \%$ à implantação de medidas de proteção individual. 
Entre as empresas que apresentaram inconsistências relacionadas com os riscos físicos, em $35,7 \%$ delas a inconsistência referia-se ao reconhecimento dos riscos, em $35,7 \%$ à avaliação quantitativa, em $60,7 \%$ à implantação de medidas coletivas e, em $17,9 \%$ à implantação de medidas de proteção individual.

\section{PCMSO: resultado das auditorias}

Os Programas de Controle Médico de Saúde Ocupacional (PCMSO) foram anali- sados, tomando-se como base os riscos à saúde dos trabalhadores, especialmente os identificados no PPRA da empresa e aqueles verificados pelos Auditores Fiscais durante a inspeção realizada no local de trabalho. Foi avaliado o cumprimento das ações e das metas previstas no planejamento anual, especialmente em relação à realização obrigatória dos exames médicos ocupacionais. Os resultados são apresentados na Tabela 02, tendo sido analisados os seguintes tópicos:

Tabela 02 PCMSO: Resultados das Auditorias.

\begin{tabular}{|l|c|c|}
\hline Procedimentos & $\begin{array}{c}\text { Sim } \\
\%\end{array}$ & $\begin{array}{c}\mathbf{N a ̃ o} \\
\%\end{array}$ \\
\hline Elaboroção do PCMSO & 93,3 & 6,7 \\
\hline Avaliação anual do PCMSO & 21,4 & 78,6 \\
\hline Inconsistências no PCMSO & 85,7 & 14,3 \\
\hline Inconsistências relacionadas com riscos físicos & 50,0 & 50,0 \\
\hline Inconsistências relacionadas com riscos cuímicos & 7,1 & 92,9 \\
\hline Inconsistências relacionadas com riscos biclógicos & 7,1 & 92,9 \\
\hline Inconsistências relacionadas com o prontuário clínico individual & 3,6 & 96,4 \\
\hline Inconsistências relacionadas com o atestado de saúde ocupacional & 17,9 & 82,1 \\
\hline Inconsistências na realização dos exames mécicos clínicos & 17,9 & 82,1 \\
\hline Inconsistências na periocicidade dos exames médicos ocupacioncis & 21,4 & 78,6 \\
\hline Inconsistências na realizaçc̃o dos exames mécicos complementares & 57,1 & 42,9 \\
\hline
\end{tabular}

\section{a. Elaboração do programa}

Todas as empresas, independente do número de empregados ou do grau de risco de suas atividades, estão obrigadas a elaborar e implementar o PCMSO, de acordo com a NR-7, item 7.1.1, da Portaria 3214/ 78. Das 30 empresas inspecionadas, 2 $(6,7 \%)$ não tinham elaborado e implementado o referido programa.

\section{b. Avaliação anual}

A NR-7, em seu item 7.4.6, estabelece que o programa deverá obedecer a um planejamento em que estejam previstas as ações de saúde a serem executadas durante o ano, devendo estas ser objeto de relatório anual. Entre as 28 empresas inspecionadas que elaboraram o PCMSO, $22(78,6 \%)$ não tinham efetuado uma avaliação anual do seu programa.

\section{c. Inconsistências identificadas}

Entende-se aqui por "inconsistência", a qualidade ou estado de falta de consistência, de fundamento ou de coerência entre os riscos à saúde dos trabalhadores e os procedimentos médicos efetivamente realizados no desenvolvimento do PCMSO. Neste sentido, foram avaliadas as seguintes etapas: identificação dos riscos ocupacionais a que está ou será exposto cada trabalhador da empresa, realização dos exames médicos ocupacionais, execução e interpretação dos indicadores biológicos, planejamento anual das ações de saúde, registro dos dados em prontuário clínico individual e emissão do Atestado de Saúde Ocupacional (ASO).

Entre as 28 empresas que elaboraram o PCMSO, $24(85,7 \%)$ delas apresentaram algum tipo de inconsistência em seu programa. As inconsistências verificadas nos programas relacionavam-se em $50,0 \%$ dos casos com o monitoramento biológico dos riscos físicos, em 7,1\% essa relação era com os riscos químicos e em $7,1 \%$ as inconsistências estavam relacionadas com os riscos biológicos presentes nos locais de trabalho. 
No caso das empresas que apresentaram inconsistências em seu PCMSO, em 57,1\% delas a inconsistência relacionava-se à realização dos exames complementares (indicadores biológicos), em $21,4 \%$ à periodicidade dos exames médicos, em $17,9 \%$ delas à realização dos exames médicos clínicos, em $17,9 \%$ à emissão do atestado de saúde ocupacional e em 3,6\% ao registro de dados em prontuário clínico individual.

Ao analisar as inconsistências, relacionando-as aos vários tipos de exames médicos ocupacionais obrigatórios, verificamos que em $71,4 \%$ das empresas a inconsistência verificada no $\mathrm{PCMSO}$ estava relacionada com o exame periódico, em 3,6\% com o exame admissional e, em 3,6\% com o exame médico demissional.

\section{O Mapa de Riscos}

O Mapa de Riscos, previsto na NR-5 da Portaria 3214/78 (1), tem por objetivo reunir as informações necessárias para estabelecer o diagnóstico da situação de segurança e saúde no trabalho na empresa. Elabo- rar o Mapa de Riscos é atribuição dos integrantes da Comissão Interna de Prevenção de Acidentes (CIPA), com a participação do maior número de trabalhadores. Na elaboração do mapa, busca-se conhecer o processo de trabalho, identificar os riscos existentes no local analisado e, ao mesmo tempo, possibilitar a troca e divulgação de informações entre os trabalhadores, bem como estimular sua participação nas atividades de prevenção. $\mathrm{O}$ conhecimento e a percepção que os trabalhadores têm do processo de trabalho e dos riscos ambientais presentes, incluindo os dados consignados no Mapa de Riscos, deverão ser considerados para fins de planejamento e execução do PPRA. Contudo, entre as 30 empresas inspecionadas, 13 (43,3\%) não tinham elaborado o Mapa de Riscos. Entre as 17 empresas que elaboraram o Mapa de Riscos, em 9 (53,0\%) essa elaboração não tinha contado com qualquer participação dos trabalhadores e em $16(94,1 \%)$ não houve a participação de representantes de seus sindicatos, conforme resultados apresentado na Tabela 03 .

Tabela 03 Mapa de Riscos: Resultados das Auditorias.

\begin{tabular}{|l|c|c|}
\hline Procedimentos & $\begin{array}{c}\text { Sim } \\
\%\end{array}$ & $\begin{array}{c}\text { Não } \\
\%\end{array}$ \\
\hline Elabcração do Mapa de Riscos & 56,7 & 43,3 \\
\hline Participação des trabalhadores na elaboração & 47,0 & 53,0 \\
\hline Participação do sindicoto dos trabalhacores no elaboração & 5,9 & 94,1 \\
\hline Apresentação do Mapo na reunic̃o da CIPA & 58,8 & 41,2 \\
\hline
\end{tabular}

\section{Fiscalização estatal: a inspeção trabalhista}

No presente trabalho, analisando as anotações registradas durante os anos de 1995 a 2002 nos Livros de Inspeção do Trabalho (LIT) das empresas estudadas, constatou-se que $8(26,7 \%)$ delas não tinham sido inspecionadas durante o referi- do período, $3(10,0 \%)$ empresas foram inspecionadas apenas 1 (uma) vez, 11 $(36,7 \%)$ foram inspecionadas 2 (duas) vezes, $3(10,0 \%)$ foram inspecionadas 3 (três) vezes, $2(6,7 \%)$ foram inspecionadas 4 (quatro) vezes e $3(10,0 \%)$ foram inspecionadas 5 (cinco) ou mais vezes durante o período. Os resultados são apresentados na Tabela 04. 
Tabela 04 PPRA /PCMSO: Fiscalização Estatal.

\begin{tabular}{|l|c|}
\hline $\begin{array}{l}\text { No de inspeções realizadas } \\
\text { no período 1995-2002 }\end{array}$ & $\%$ de empresas \\
\hline Nenhuma & 26,7 \\
\hline 01 & 10,0 \\
\hline 02 & 36,7 \\
\hline 03 & 10,0 \\
\hline 04 & 6,7 \\
\hline 05 ou mais & 10,0 \\
\hline
\end{tabular}

Após inspecionar as 30 empresas selecionadas, auditando seus programas PPRA e PCMSO, foram emitidos 29 Termos de Notificação (TN) para que as empresas regularizassem 215 itens, perfazendo uma média de 7 irregularidades por empresa.

\section{Controle social e a participação dos trabalhadores}

A experiência mundial tem demonstrado que a ação da fiscalização estatal é limitada e raramente tem sido suficiente, o que torna cada vez mais importante o controle social, isto é, a fiscalização exercida diretamente pelos próprios trabalhadores e pelos seus sindicatos. De acordo com a NR9 , itens 9.4 e 9.5, os trabalhadores interessados têm o direito de apresentar propostas e receber informações e orientações a fim de assegurar a proteção aos riscos ambientais identificados na execução do PPRA. Além disso, a norma estabelece que colaborar e participar na implantação e na execução do PPRA é uma das responsabilidades dos trabalhadores.

Os documentos-base e os relatórios anuais do PPRA e do PCMSO, por sua vez, devem ser apresentados e discutidos na Comissão Interna de Prevenção de Acidentes (CIPA). A implantação de medidas de caráter coletivo deve ser sempre acompanhada de treinamento dos trabalhadores, quanto aos procedimentos que assegurem a sua eficiência, e de informação sobre as eventuais limitações de proteção que ofereçam. O trabalhador tem também o direito de receber cópia dos resultados dos procedimentos médicos a que foi submetido, além da segunda via do seu Atestado de Saúde Ocupacional (ASO). Convém destacar, finalmente, que ao estabelecer os parâmetros mínimos e as diretrizes gerais a serem observados na execução dos programas PPRA e PCMSO, as normas estabelecem, também, que os mesmos podem ser ampliados mediante negociação coletiva de trabalho.

No presente estudo, entre as 28 empresas que tinham elaborado o PPRA ou 0 PCMSO, em nenhuma delas essa elaboração tinha contado com qualquer participação dos trabalhadores (ou de representantes de seu sindicato), sendo que em 25 $(89,3 \%)$ os documentos-base do PPRA ou do PCMSO não foram apresentados e discutidos na Comissão Interna de Prevenção de Acidentes (CIPA). A ampliação das diretrizes gerais e dos parâmetros mínimos dos programas PPRA e PCMSO, mediante negociação coletiva de trabalho, também não foi verificada entre as empresas inspecionadas. Os resultados relacionados com a avaliação do controle social são apresentados na Tabela 05. 
Tabela 05 PPRA / PCMSO: Controle Social.

\begin{tabular}{|l|c|c|}
\hline Procedimentos & $\begin{array}{c}\text { Sim } \\
\%\end{array}$ & $\begin{array}{c}\text { Não } \\
\%\end{array}$ \\
\hline $\begin{array}{l}\text { Participação dos trabalhodores (ou de seus sindicatos) no } \\
\text { desenvolvimento do PPRA ou do PCMSO }\end{array}$ & 0,0 & 100,0 \\
\hline $\begin{array}{l}\text { Ampliação dos parâmetros do PPRA ou do PCMSO mediante } \\
\text { negaciação coletiva de trabalho }\end{array}$ & 0,0 & 100,0 \\
\hline \begin{tabular}{l} 
Apresentação do PPRA ou do PCMSO na reunião da CIPA \\
\hline
\end{tabular} & 10,7 & 89,3 \\
\hline
\end{tabular}

\section{Conclusões}

A legislação brasileira de segurança e saúde no trabalho, a partir do final de 1994, passou a adotar um novo enfoque e estabeleceu a obrigatoriedade das empresas elaborarem e implementarem dois programas: um ambiental, o PPRA - Programa de Prevenção de Riscos Ambientais, e outro médico, o PCMSO - Programa de Controle Médico de Saúde Ocupacional. As novas normas privilegiaram o instrumental clínico-epidemiológico na abordagem da relação saúde/trabalho e introduziram a questão da valorização da participação dos trabalhadores e do controle social.

Contudo, ao inspecionar 30 empresas baianas com mais de 100 empregados, os Autores evidenciaram que $2(6,7 \%)$ delas não tinham elaborado o PPRA ou o PCMSO. Entre as 28 empresas que elaboraram o PPRA, 92,9\% delas apresentaram algum tipo de inconsistência em seu programa, sendo que $85,7 \%$ não tinham efetuado uma avaliação global do PPRA. Entre as 28 empresas que elaboraram o PCMSO, 85,7\% apresentaram algum tipo de inconsistência em seu programa, sendo que $78,6 \%$ não tinham efetuado uma avaliação anual do PCMSO.

Após um período de 8 anos de vigência da legislação que introduziu os programas PPRA e PCMSO, os Autores constataram que $26,7 \%$ das empresas estudadas não tinham sido inspecionadas durante o referido pe- ríodo, sendo que $83,4 \%$ das empresas foram inspecionadas 3 (três) vezes ou menos. Em relação ao controle social, isto é, a fiscalização exercida diretamente pelos próprios trabalhadores e pelos seus sindicatos, foi possível evidenciar que entre as empresas que elaboraram o PPRA ou o PCMSO, em nenhuma delas essa elaboração tinha contado com participação dos trabalhadores ou do sindicato profissional. A ampliação das diretrizes gerais e dos parâmetros mínimos dos programas PPRA e PCMSO, mediante negociação coletiva de trabalho, também não foi verificada entre as empresas inspecionadas.

Concluindo, apesar das novas normas privilegiarem o instrumental clínicoepidemiológico e valorizarem a participação dos trabalhadores e o controle social, no presente trabalho foi possível constatar a baixa qualidade técnica dos programas PPRA e PCMSO, a ação limitada e insuficiente da fiscalização estatal dos ambientes de trabalho, além do precário controle social, isto é, a inexistência de uma fiscalização exercida diretamente pelos próprios trabalhadores e pelos seus sindicatos. Os Autores apontam a evidente necessidade de ampliar a cobertura da fiscalização estatal, estimular a participação dos trabalhadores e dos seus representantes no desenvolvimento dos programas PPRA e PCMSO e, desenvolver e aprimorar condutas, procedimentos e instrumentos de inspeção na área de segurança e saúde no trabalho. 


\section{Referências Bibliográficas}

1. MTE - MINISTÉRIO DO TRABALHO E EMPREGO. SSST - Secretaria de Segurança e Saúde no Trabalho. Legislação de Segurança e Saúde no Trabalho. Brasilia, 1999.

2. OIT - ORGANIZAÇÃO INTERNACIONAL DO TRABALHO. Conferência Internacional do Trabalho (70 reunião). Serviços de saúde dos trabalhadores. Informe IV(2). Genebra: OIT, 1985.

3. Moura, M.A. - Um olhar coletivo. Revista Proteção, 40-43, maio de 1998.

4. Miranda, C.R. - Inspeção do Trabalho, Epidemiologia e Segurança e Saúde no Trabalho. In: A importância da Inspeção do Trabalho - Trabalhos Premiados. Brasília (DF) : Sindicato Nacional dos Agentes da Inspeção do Trabalho (SINAIT), 1999.

5. Pena, P.L.G. - Elementos teóricos e metodológicos para a elaboração do PPRA e do PCMSO. FAMED/UFBA. Salvador, julho 2000 (mimeo).

6. Saad, I.F.S.D. \& Giampaoli, E. Programa de Prevenção de Riscos Ambientais - NR-9 Comentada. $4^{\circ}$ edição. ABHO: São Paulo, 1999.

7. MTE - MINISTÉRIO DO TRABALHO E EMPREGO. SSST - Secretaria de Segurança e Saúde no Trabalho.
Norma Regulamentadora $\mathrm{n}^{\circ}$ 7: nota técnica. Brasília: MTb, SSST, 1996. 34 p.

8. DAL ROSSO, S. - A Inspeção do Trabalho - Capítulo 9 do Livro "O Trabalho na Sociedade. O Castigo de Prometeu". Brasília (DF) : Sindicato Nacional dos Agentes da Inspeção do Trabalho (SINAIT), $1997 . \quad 42 p$.

9. OIT - ORGANIZAÇÃO INTERNACIONAL DO TRABALHO. Convenção no 81 sobre a Inspeccão do Trabalho. Genebra : OIT, 1947.

10. SISTEMA FEDERAL DE INSPEÇÃO DO TRABALHO-SFIT. Quadro de Auditores Fiscais do Trabalho. Brasília : Ministério do Trabalho e Emprego (MTE), dezembro de 2002.

11. INSTITUTO BRASILEIRO DE GEOGRAFIA E ESTATÍSTICAS - IBGE. Diretoria de Pesquisas, Divisão de Cadastro e Classificação. Cadastro Central de Empresas. Brasília, 1999.

12. SISTEMA FEDERAL DE INSPEÇÃO DO TRABALHO - SFIT. Ministério do Trabalho e Emprego (MTE). Consulta parametrizada de empresas: áreas da Indústria (CNAE: 15.11-3 a 36.99-4), Comércio (CNAE: 50.10-5 a 52.79-5) e Serviços (CNAE: 55.11-5 a 93.092), com 100 ou mais empregados (Faixa=19 a 37), setembro de 2002. 